\title{
Short-term effect of the COVID-19 pandemic on suicidal ideation: A prospective cohort study
}

\author{
Hajime Sueki ${ }^{1 *} \&$ Michiko Ueda ${ }^{2}$
}

\begin{abstract}
Background: Few studies have examined the effect of pandemics on suicide-related outcomes. Aims: We examined whether suicidal ideation levels among the general population changed owing to the COVID-19 pandemic by tracking individuals between January and April 2020. Method: We used a prospective observational longitudinal design $(n=6,683)$ to conduct online surveys of the general adult population in Japan before (baseline) and during the pandemic (follow-up). Results: Suicidal ideation levels were significantly lower during than before the pandemic; however, the effect size was small $(r=.07)$. Participants who were younger, with unstable employment, without children, with low income, and receiving psychiatric care were more likely to have higher suicidal ideation levels during the pandemic. Limitations: The dropout rate may have affected the results. COVID-19 cases and deaths in Japan were relatively lower than in other developed countries. Conclusion: Although the short-term impact of COVID-19 on suicidal ideation is limited, relatively young and economically vulnerable individuals are more likely to show exacerbated suicidal ideation during the pandemic.
\end{abstract}

\section{Keywords}

Suicide prevention; suicidal behaviors; mental health; public health; novel coronavirus

1 Faculty of Human Sciences, Wako University, 5-1-1 Kanaigaoka, Machida, Tokyo, 195-8585, Japan 2 Faculty of Political Science and Economics, Waseda University, Building No.3 1-6-1 Nishiwaseda, Shinjuku-ku, Tokyo, 169-8050, Japan

* Corresponding author: Hajime Sueki, Faculty of Human Sciences, Wako University, 5-1-1 Kanaigaoka, Machida, Tokyo, 195-8585, Japan, h_sueki@wako.ac.jp, Tel: +81-44-989-7777. 


\section{Introduction}

The 2020 COVID-19 pandemic is an unprecedented public health crisis that has substantially disrupted economic and social activities. Approximately 22.6 million people worldwide have been infected and 790,000 have died from COVID-19 (Worldometers, 2020). To reduce the spread of SARS-CoV-2, many countries have adopted social and physical distancing policies (e.g., local/national movement restrictions, stay-at-home orders, closure of non-essential businesses/services). These policies may help to prevent the spread of the virus, but they also have large-scale economic and psychosocial ramifications. Reduced economic activity has led to economic decline in many countries, and social distancing and stay-at-home measures can cause psychological distress. These factors may have adverse effects on public mental health. Indeed, studies in various countries show that the general population's mental health has worsened since the pre-pandemic period (Pierce et al., 2020; Salari et al., 2020). Because economic shocks and deteriorating mental health status can be major suicide risk factors, the number of suicides may rise during and after the pandemic (Gunnell et al., 2020; Reger et al., 2020).

However, the effect of pandemics on suicide remains unclear. There are few high-quality studies on the effect of previous pandemics (e.g., the 1918-1919 great influenza epidemic, severe acute respiratory syndrome (SARS)) on suicide (Niederkrotenthaler et al., 2020; Zortea et al., 2020), although some studies suggest that suicides tend to increase after pandemics (Chan et al., 2006; Cheung et al., 2008; Honigsbaum, 2010; Wasserman, 1992; Yip et al., 2010). However, to the best of our knowledge, there are no peer-reviewed studies on the effect of the COVID-19 pandemic on suicide and suicidal ideation, although some evidence is emerging in preprints. A Peruvian study showed that violent and accidental deaths, including suicide, declined during the COVID-19 lockdown period (Calderon-Anyosa \& Kaufman, 2020). A U.S. Internet survey reported that COVID-19-related experiences are associated with suicidal thoughts and behaviors (Ammerman et al., 2020). However, the latter cross-sectional study used a convenience sample and lacked pre-COVID-19 data.

It is also unclear which populations are most likely to be adversely affected by pandemics. The suicide rate of elderly people in Hong Kong increased after the SARS pandemic (Chan et al., 2006; Cheung et al., 2008), but the findings may not be generalizable to the COVID-19 pandemic because the societal effects of COVID-19 are much larger and more wide-ranging than those of any other recent pandemics. To develop clear, effective suicide prevention policies for pandemics (Niederkrotenthaler et al., 2020), it is important to identify and target individuals likely to be at increased risk of suicide in a pandemic.

The study aim was to compare suicidal ideation levels of the general population in Japan during the pandemic to that of the pre-pandemic period, and to identify those individuals experiencing suicidal ideation during the pandemic. To examine changes in suicidal ideation, we used a longitudinal survey to measure suicidal ideation immediately before the COVID-19 pandemic and in the middle of the first wave of the pandemic in Japan. 


\section{Methods}

\section{Study design and sampling}

We used a prospective observational longitudinal design. Baseline (T1) and follow-up (T2; approximately three months after T1) surveys were conducted in Japan with members of Internet survey panels through a major Japanese Internet survey company (Cross Marketing Inc., Tokyo, Japan). Japan ranks 45th in the number of infected cases. As of August 2020, there were 57,600 registered cases and 1,128 deaths (Worldometers, 2020), or 8.91 deaths per one million persons. Figure 1 shows the timing of the surveys and the monthly number of new COVID-19 cases in Japan. The T1 survey was conducted on January 24, 2020, when there were only two COVID-19 cases in Japan. The T2 survey was conducted between April 27 and 30, 2020. The number of COVID-19 cases in Japan began to increase in March, and the government declared a state of emergency on April 7. The reported COVID-19 cases were mainly in urban areas (Tokyo, Kanagawa, Saitama, Chiba, Osaka, Hyougo, Fukuoka), which were designated special alert areas when the state of emergency was declared. The cases in April constituted the first wave of infections and corresponded to the COVID-19 epidemic period.

Figure 2 shows the sampling process. The T1 survey was based on a target population aged 20 years or older and distributed according to the demographics of the latest Japanese census data (Japanese Ministry of Internal Affairs and Communications, 2016). The stratified variables were age, sex, and geographic area of residence. We distributed questionnaires to 125,011 individuals selected according to the stratified variables. Of these, 15,698 individuals accessed the survey website and 9,982 completed the questionnaire at $\mathrm{T} 1$. We then invited all participants who had completed the $\mathrm{T} 1$ survey to participate in the T2 survey; 6,683 participants completed the T2 questionnaire. We analyzed T1 and T2 survey responses for these 6,683 participants.

We were unable to obtain a priori institutional review board approval because the institution to which the first author belongs has no such board. However, the study was conducted in accordance with the Helsinki Declaration (revised 1989). Prior to participation, potential participants were advised that answering some of the survey questions may lead to a mood change and that they were allowed to quit at any point during the survey. We obtained explicit informed consent from the participants online. Participants were also provided with website links containing professional support resources while they were completing the questionnaire.

\section{Measurements}

All participants completed an online self-administered questionnaire assessing suicidal ideation, other suicide-related items (e.g., current psychiatric care), and demographic details (sex, age, educational background, employment, marital status, family structure, annual household income, and residential area).

Suicidal ideation was measured using the short form of the suicidal ideation scale (Sueki, 2019) at T1 and T2. This 6-item instrument (example item: Do you wish to die?) was designed to quantify suicidal ideation. Responses are rated on a 3-point scale from 0 to 2; higher scores indicate 
increased suicidal ideation levels (total score range: $0-12$ ). The scale has shown internal consistency (Cronbach's $\alpha=.89$ ), test-retest reliability (4-week interval: Spearman's $\rho=.84$ ), and validity in a survey with 2,486 Internet users (Sueki, 2019).

\section{Statistical analyses}

To examine changes in suicidal ideation between the pre-COVID-19 (T1) and COVID-19 periods (T2), we conducted a paired $t$-test with time of measurement as the independent variable and suicidal ideation as the dependent variable. To identify vulnerable populations experiencing suicidal ideation during the pandemic, we used forced-entry multivariate regression analysis to predict suicidal ideation at T2. The model independent variables were suicidal ideation at T1, sex, age, educational background, employment, marital status, family structure, annual household income, residential area, and current psychiatric care status. Regression coefficients (B) and their 95\% confidence intervals (CI), and standardized regression coefficients $(\beta)$, were calculated. All analyses were conducted separately with data from all participants, non-suicidal group only (T1 suicidal ideation $=0)$, and suicidal group only (T1 suicidal ideation $>0$ ). Presented $p$-values are for two-tailed tests. Analyses were performed using SPSS (SPSS 24.0 for Windows; SPSS Inc., Chicago, IL, USA).

\section{Results}

\section{Participant characteristics and scale scores}

Table 1 summarizes participant characteristics. Data for 6,683 participants were analyzed; $51.2 \%$ were men and the mean age was 46.5 years $(\mathrm{SD}=13.8)$. About half of participants $(50.3 \%)$ had a university or graduate degree, were married (56.5\%), had children (50.0\%), and resided in a special alert area $(50.8 \%)$. Of participants, $41 \%$ were permanent employees, $19.8 \%$ were part-time or temporary workers, $5.7 \%$ were self-employed, $14.8 \%$ were full-time homemakers, and $12.3 \%$ were not in the labor market. The median annual household income was JPY 400-800 million, and $11.1 \%$ of participants had visited a psychiatric clinic at the time of the survey. The only missing values in the final data set were for income (participants were allowed to skip this question); respondents who did not answer this question were included in the analysis as "Income unknown."

\section{Changes in suicidal ideation}

Suicidal ideation was significantly lower during the pandemic $(M=1.59)$ than before it $(M=1.71)$, $t(6682)=5.87, p<.001$; however, the effect size was very small, $r=.07$. A paired $t$-test on data from the non-suicidal group showed that suicidal ideation was significantly higher during the pandemic ( $M$ $=0.31)$ than before it $(M=0), t(3517)=20.27, p<.001, r=.32$. Similarly, a paired $t$-test on data from the suicidal group showed that suicidal ideation was significantly lower during the pandemic $(M=$ 3.02) than before it $(M=3.61), t(3164)=15.77, p=.005, r=.27$. 


\section{Predictors of suicidal ideation at $\mathrm{T} 2$}

Multiple linear regression analysis to predict suicidal ideation at $\mathrm{T} 2$ produced a significant regression equation, $F(22,6660)=521.6, p<.001$ ), with an $R^{2}$ of .63 (Table 2). Individuals in their 30s, those with unstable employment status (part-time, temporary worker), those without children, those with relatively low annual household income ( $<$ JPY 400 million), and those currently receiving psychiatric care were more likely to have a significantly higher suicidal ideation level at T2 than the reference groups, after controlling for suicidal ideation at T1. In the non-suicidal group, relatively younger age (20-40s), low educational level, and current psychiatric care were significant predictors of suicidal ideation at $\mathrm{T} 2, F(21,3496)=3.71, p<.001, R^{2}=.02$. In the suicidal group, unstable employment status (part-time, temporary worker), annual household income ( $<$ JPY 400 million), and marital status (divorced) were significant predictors of suicidal ideation at T2, $F(22,3142)=169.5, p<.001, R^{2}$ $=.54$.

\section{Discussion}

\section{Main findings}

We used a longitudinal design to examine whether COVID-19 affected suicidal ideation levels among the general population. We found a significant reduction in suicidal ideation in April 2020, when Japan had the first wave of infection and a state of emergency was declared, compared with January 2020, when there were almost no COVID-19 cases in Japan. This finding is consistent with the number of suicides: data from the Japanese National Police Agency (2020) show that suicide numbers in January $2020(n=1,678)$ were unchanged from those in January $2019(n=1,684)$, whereas suicide numbers in April $2020(n=1,487)$ dropped to approximately 82\% of those in April $2019(n=1,814)$ (Figure $1)$.

Individuals who experienced increased suicidal ideation during the pandemic were in their 30 s, in precarious employment, with low household income, had no children, and were receiving psychiatric care. Japan's gross domestic product fell $27.8 \%$ on an annualized basis in April-June, as economic activity stalled owing to the state of emergency (Japanese Cabinet Office, 2020). This worsening economic environment is likely to have exacerbated suicidal ideation among relatively young (20-40 years) and low-income individuals in precarious employment. The absence of children was also associated with worsening suicidal ideation. During the state of emergency, people were asked to spend more time at home, although there was no strict movement restriction. Individuals with no children are more likely to live alone, so some may have spent most of the emergency period at home alone. Our findings suggest that such individuals may have experienced increased suicidal ideation.

Comparison of the groups with/without suicidal ideation before the pandemic showed differences in the characteristics of those individuals more adversely affected. In participants with no suicidal ideation before the pandemic, suicidal ideation was worse among relatively young individuals attending psychiatric hospitals. However, in participants with suicidal ideation before the pandemic, unstable employment and low household income contributed to worsening suicidal ideation. During 
the pandemic, people with mental health vulnerabilities may be most likely to have increased suicidal ideation, followed by those with economic instability. For groups at particularly high risk of suicide, implementing financial support alongside mental health treatment may be important in reducing suicide risk.

\section{Comparison with previous studies}

Several studies, including one in Japan (Ueda et al., 2020), indicate that the general population's mental health may have deteriorated during the COVID-19 pandemic (Salari et al., 2020); however, the present findings seem to contradict these previous results. Durkheim's theory that increased social integration reduces suicide in society as a whole may provide a possible explanation. The current pandemic crisis requires efforts by all members of society, and working towards a common goal may have increased social cohesion levels. Although no Japanese study has investigated whether people have experienced changes in social connectedness during the pandemic, a study in the United Kingdom in June 2020 found that 47\%, 45\%, and 31\% of survey respondents felt more connected with their family members, neighbors, and local community, respectively, during lockdown (Abrams et al., 2020). Studies on the effect of natural and human-induced disasters on suicide (Matsubayashi et al., 2013; Orui et al., 2018; Safarpour et al., 2020) show that suicide rates tend to rise after a large disaster (Safarpour et al., 2020), but can temporarily drop after a natural disaster, as long as the scale of the disaster is not very large (Matsubayashi et al., 2013). This is because social integration tends to increase after natural disasters, which can protect against suicide risk (Matsubayashi et al., 2013). Thus, greater social integration during this pandemic may have counteracted the effect of deteriorating mental health, preventing vulnerable individuals from developing suicidal ideation. However, previous disaster studies also suggest that the reduction in suicide numbers in Japan may be shortly followed by an increase. Indeed, suicide numbers returned to their usual level in July 2020 (Figure 1), which calls for careful monitoring in the upcoming months.

Importantly, some groups are experiencing higher suicidal ideation levels during the pandemic, although the overall suicidal ideation level has slightly decreased. Individuals whose mental health has deteriorated during the pandemic and those whose suicidal ideation has worsened share many similarities. Mental health during this pandemic is worse among younger people (Ahmed et al., 2020; Salari et al., 2020; Ueda et al., 2020), although older people have a higher mortality rate from COVID-19 infection. Unemployment or precarious employment for self/family also contribute to poor mental health (Cao et al., 2020; Ueda et al., 2020). Young age and unstable employment were associated with increased suicidal ideation in this study. However, the presence of close others, such as living with family members, may protect against poor mental health (Cao et al., 2020), which is consistent with our finding that the presence of children protects against suicidal ideation. There is an urgent need to implement a system of care for these at-risk groups, particularly if suicide numbers begin to rise in the near future. 


\section{Strengths and limitations}

This is the first prospective cohort study to examine the effect of a pandemic on suicidal ideation. A major study strength is that suicidal ideation was measured before the pandemic. To the best of our knowledge, no longitudinal studies have tracked suicidal ideation levels from pre-pandemic to pandemic periods.

Study limitations include the possibility that participants who became suicidal during the pandemic were more likely to have been excluded from the T2 survey. Of the 9,982 participants who completed the T1 survey, approximately $33 \%$ did not complete the T2 survey. If individuals whose suicidal ideation increased after the T1 survey tended to drop out of the T2 survey, this could have resulted in a reduction in suicidal ideation at T2. However, there was no discernible difference in demographic characteristics between the T1 and T2 respondents. Additionally, the generalization of results requires caution. This was a relatively short-term longitudinal survey. At the time of the study, COVID-19 infections and deaths in Japan were relatively lower than in other developed countries (Worldometers, 2020). It is unclear whether the present findings can be generalized to severely affected areas, as disaster severity can affect subsequent variations in suicide rates (Matsubayashi et al., 2013).

\section{Conclusions and future research}

Despite its limitations, this is the first longitudinal study to track suicidal ideation levels before and during the COVID-19 pandemic. Although the short-term effect of the pandemic on suicidal ideation was limited for the whole sample, relatively young and economically vulnerable people showed a higher risk of developing suicidal ideation during the pandemic. Pandemics may have a long-term effect on suicide risks (Chan et al., 2006; Cheung et al., 2008; Honigsbaum, 2010; Wasserman, 1992; Yip et al., 2010); thus, additional research is needed to understand the long-term consequences of the current pandemic and to protect vulnerable individuals. 


\section{Acknowledgements}

This work was financially supported by JSPS Grants-in-Aid for young scientists Grant Number 18K13358 and JSPS Grants-in-Aid for Scientific Research Grant Number 20H01584. The funders had no role in study design, data collection and analysis, decision to publish, or preparation of the manuscript. 


\section{References}

Abrams, D., Lalot, F., Broadwood, J., \& Platts-Dunn, I. (2020). Perceptions of COVID-19 and social cohesion (July 2020 Report). https://mk0nuffieldfounpg9ee.kinstacdn.com/wpcontent/uploads/2020/04/Abrams-community-cohesion-report-July-2020.pdf

Ahmed, M. Z., Ahmed, O., Aibao, Z., Hanbin, S., Siyu, L., \& Ahmad, A. (2020). Epidemic of COVID19 in China and associated psychological problems. Asian Journal of Psychiatry, 51, 102092. https://doi.org/10.1016/j.ajp.2020.102092

Ammerman, B. A., Burke, T. A., Jacobucci, R., \& McClure, K. (2020). Preliminary investigation of the association between COVID-19 and suicidal thoughts and behaviors in the U.S. PsyArXiv. https://doi.org/10.31234/osf.io/68djp

Calderon-Anyosa, R. J., \& Kaufman, J. S. (2020). Impact of COVID-19 lockdown policy on homicide, suicide, and motor vehicle deaths in Peru. medRxiv. https://doi.org/10.1101/2020.07.11.20150193

Cao, W., Fang, Z., Hou, G., Han, M., Xu, X., Dong, J., \& Zheng, J. (2020). The psychological impact of the COVID-19 epidemic on college students in China. Psychiatry Research, 287, 112934. https://doi.org/10.1016/j.psychres.2020.112934

Chan, S. M. S., Chiu, F. K. H., Lam, C. W. L., Leung, P. Y. V., \& Conwell, Y. (2006). Elderly suicide and the 2003 SARS epidemic in Hong Kong. International Journal of Geriatric Psychiatry, 21, 113-118. https://doi.org/10.1002/gps. 1432

Cheung, Y. T., Chau, P. H., \& Yip, P. S. (2008). A revisit on older adults suicides and severe acute respiratory syndrome (SARS) epidemic in Hong Kong. International Journal of Geriatric Psychiatry, 23, 1231-1238. https://doi.org/10.1002/gps.2056

Gunnell, D., Appleby, L., Arensman, E., Hawton, K., John, A., Kapur, N., Khan, M., O’Connor, R. C., Pirkis, J., Appleby, L., Arensman, E., Caine, E. D., Chan, L. F., Chang, S. S., Chen, Y. Y., Christensen, H., Dandona, R., Eddleston, M., Erlangsen, A., .. Yip, P. S. (2020). Suicide risk and prevention during the COVID-19 pandemic. The Lancet Psychiatry, 7(6), 468-471. https://doi.org/10.1016/S2215-0366(20)30171-1

Honigsbaum, M. (2010). The great dread: Cultural and psychological impacts and responses to the 'Russian' influenza in the United Kingdom, 1889-1893. Social History of Medicine, 23, 299319. https://doi.org/10.1093/shm/hkq011

Japanese Cabinet Office (2020). Flash report of quarterly GDP. https://www.esri.cao.go.jp/jp/sna/sokuhou/sokuhou_top.html

Japanese Ministry of Internal Affairs and Communications. (2016). 2015 census. https://www.stat.go.jp/data/kokusei/2015/kekka.html

Japanese National Police Agency (2020). The number of suicides. https://www.npa.go.jp/publications/statistics/safetylife/jisatsu.html

Matsubayashi, T., Sawada, Y., \& Ueda, M. (2013). Natural disasters and suicide: Evidence from Japan. Social Science \& Medicine, 82, 126-133. https://doi.org/10.1016/j.socscimed.2012.12.021 
Niederkrotenthaler, T., Gunnell, D., Arensman, E., Pirkis, J., Appleby, L., Hawton, K., ... \& Platt, S. (2020). Suicide research, prevention, and COVID-19: Towards a global response and the establishment of an international research collaboration. Crisis. https://doi.org/10.1027/02275910/a000731

Orui, M., Suzuki, Y., Maeda, M., \& Yasumura, S. (2018). Suicide rates in evacuation areas after the Fukushima Daiichi nuclear disaster. Crisis, 39, 353-363. https://doi.org/10.1027/02275910/a000509

Pierce, M., Hope, H., Ford, T., Hatch, S., Hotopf, M., John, A., Kontopantelis, E., Webb, R., Wessely, S., McManus, S., \& Abel, K. M. (2020). Mental health before and during the COVID-19 pandemic: A longitudinal probability sample survey of the UK population. The Lancet Psychiatry, S2215036620303084. https://doi.org/10.1016/S2215-0366(20)30308-4

Reger, M. A., Stanley, I. H., \& Joiner, T. E. (2020). Suicide mortality and coronavirus disease 2019-A perfect storm? JAMA Psychiatry. https://doi.org/10.1001/jamapsychiatry.2020.1060

Safarpour, H., Sohrabizadeh, S., Malekyan, L., Safi-Keykaleh, M., Pirani, D., Daliri, S., \& Bazyar, J. (2020). Suicide death rate after disasters: A meta-analysis study. Archives of Suicide Research. https://doi.org/10.1080/13811118.2020.1793045

Salari, N., Hosseinian-Far, A., Jalali, R., Vaisi-Raygani, A., Rasoulpoor, S., Mohammadi, M., ... \& Khaledi-Paveh, B. (2020). Prevalence of stress, anxiety, depression among the general population during the COVID-19 pandemic: A systematic review and meta-analysis. Globalization \& Health, 16, 1-11. https://doi.org/10.1186/s12992-020-00589-w

Sueki, H. (2019). Tanshukuban zisatsu nenryo shakudo no sakusei [Development of a short form of the suicidal ideation scale]. Suicide Prevention \& Crisis Intervention, 39(2), 94-101.

Ueda, M., Stickley, A., Sueki, H., \& Matsubayashi, T. (2020). Mental health status of the general population during the COVID-19 pandemic. Psychiatry \& Clinical Neurosciences. doi:10.1111/pcn.13105

Wasserman, I. M. (1992). The impact of epidemic, war, prohibition and media on suicide: United States, 1910-1920. Suicide \& Life-Threatening Behavior, 22, 240-254. https://doi:10.1111/j.1943-278X.1992.tb00231.x

Worldometers. (2020). COVID-19 coronavirus pandemic. https://www.worldometers.info/coronavirus/

Yip, P. S., Cheung, Y. T., Chau, P. H., \& Law, Y. W. (2010). The impact of epidemic outbreak: The case of severe acute respiratory syndrome (SARS) and suicide among older adults in Hong Kong. Crisis, 31, 86-92. https://doi.org/10.1027/0227-5910/a000015

Zortea, T. C., Brenna, C. T. A., Joyce, M., McClelland, H., Tippett, M., Tran, M., \& Platt, S. (2020). The impact of infectious disease-related public health emergencies on suicide, suicidal behavior, and suicidal thoughts: A systematic review. https://covid19mentalhealthresearch.ca/wp-content/uploads/2020/06/HATCHER-InitialKnowledge-Synthesis-2020-06-22.pdf. 


\section{The number of}

suicide deaths

State of emergency

The number of

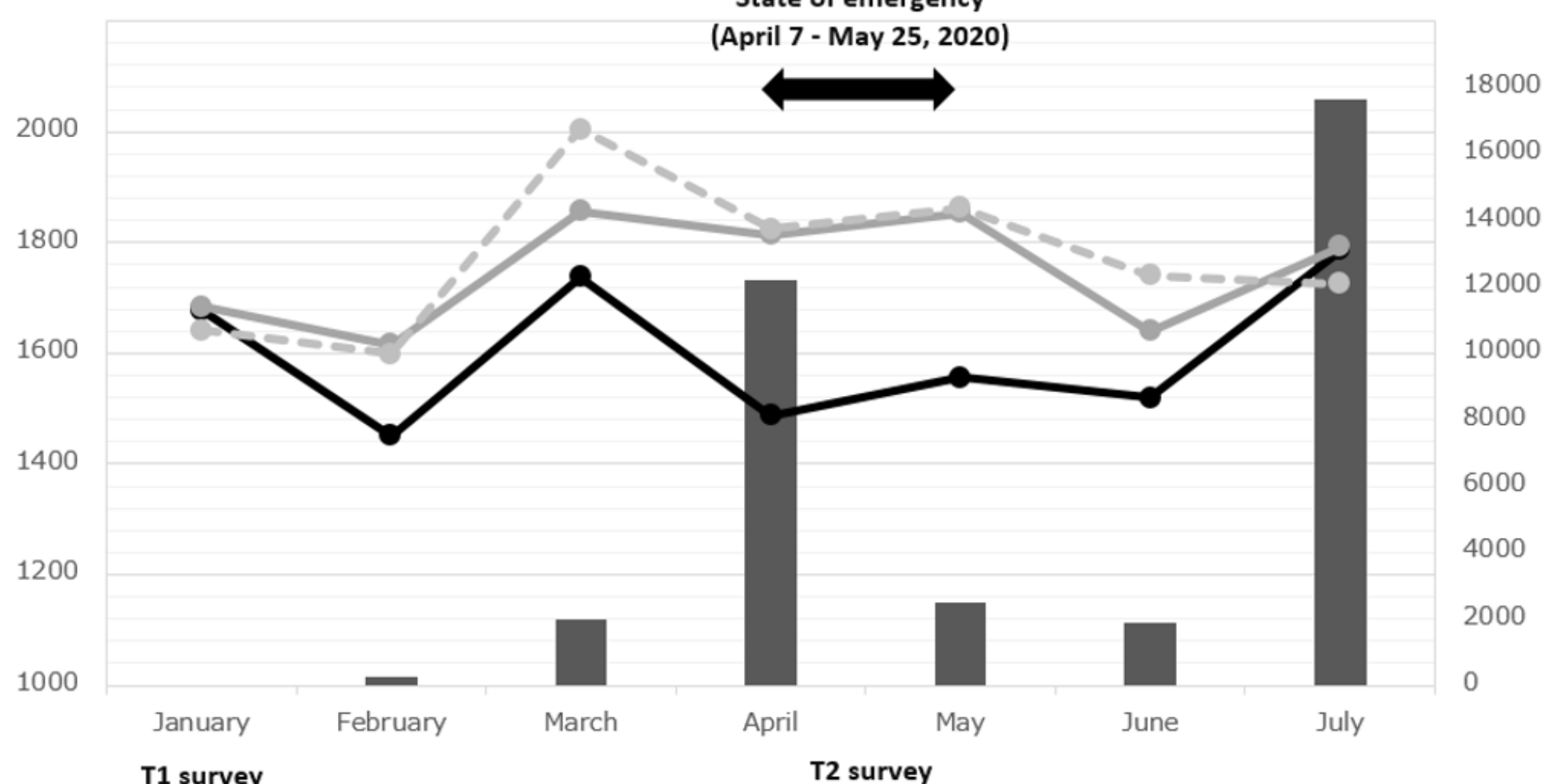

T1 survey

January 24, 2020

April 27 - April 30, 2020

Figure 1 Monthly COVID-19 infections and suicide deaths in Japan 


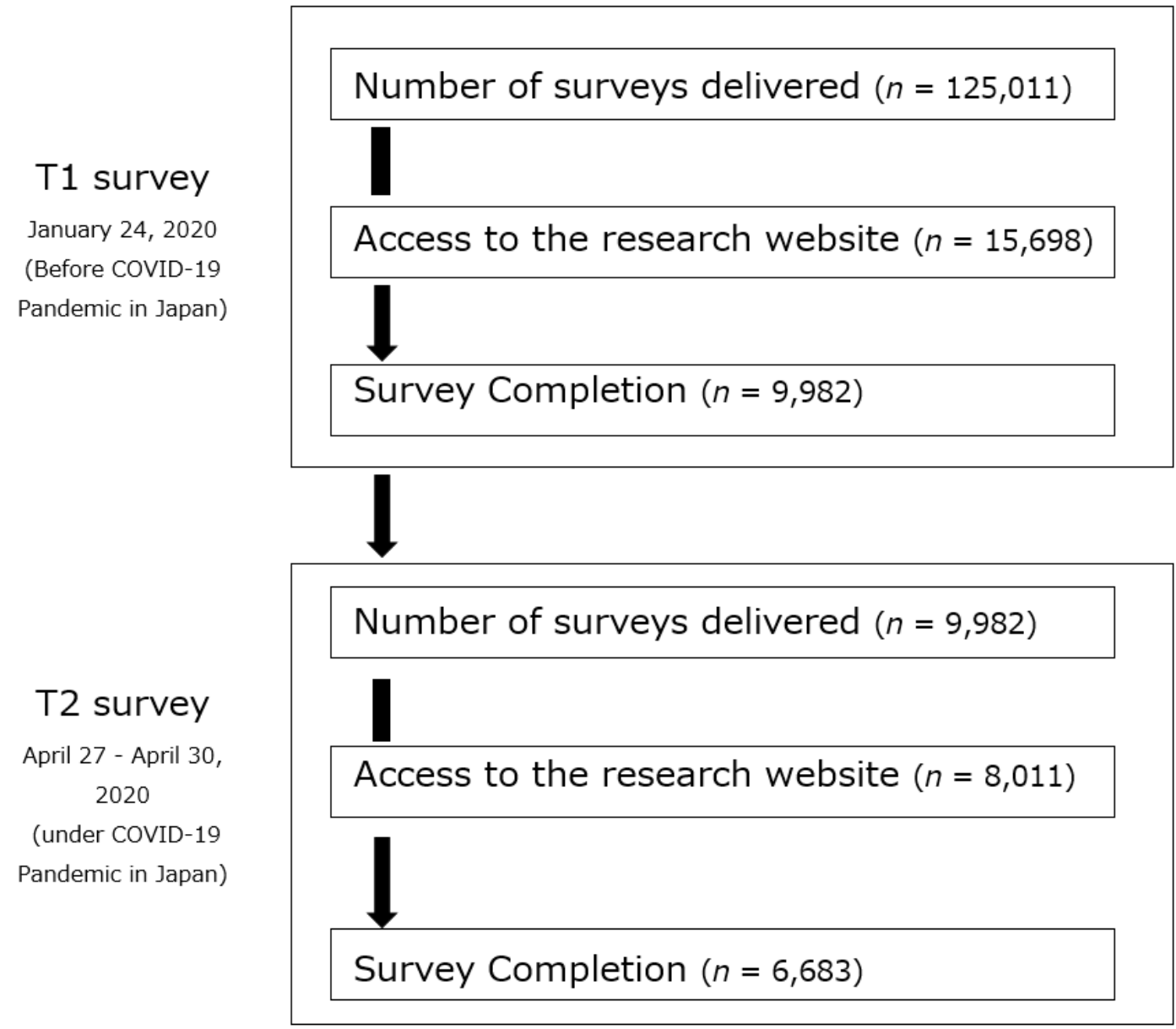

Figure 2 Research design 
Table 1 Participant characteristics

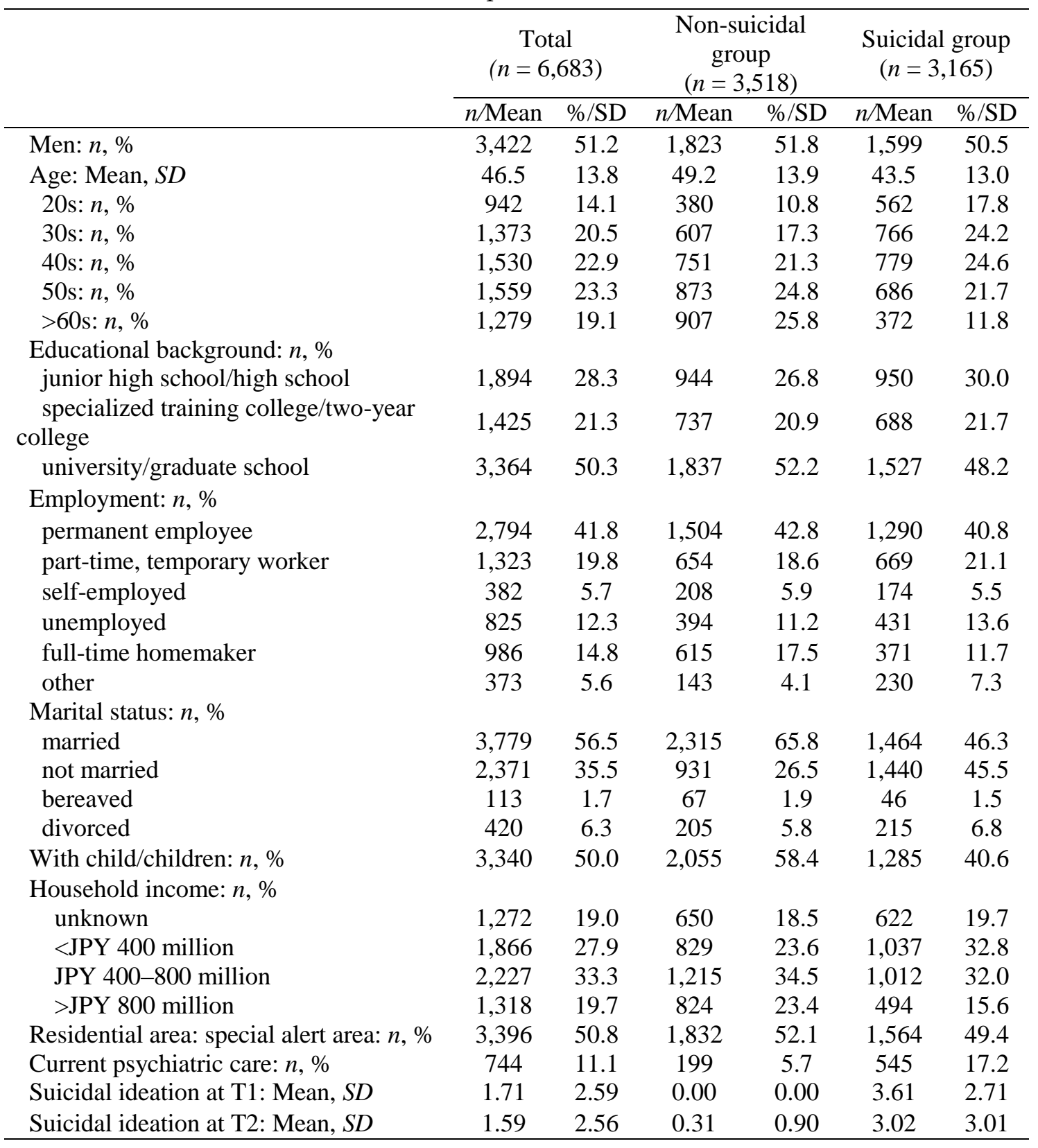


Table 2 Multiple regression analysis: suicidal ideation (T2) as the dependent variable

\begin{tabular}{|c|c|c|c|c|c|c|c|c|c|c|c|c|c|c|c|}
\hline & \multicolumn{5}{|c|}{ Total } & \multicolumn{5}{|c|}{ Non-suicidal group } & \multicolumn{5}{|c|}{ Suicidal group } \\
\hline & \multirow{2}{*}{$B$} & \multicolumn{2}{|c|}{$95 \%$ CI } & \multirow{2}{*}{$\beta$} & \multirow[b]{2}{*}{$p$} & \multirow{2}{*}{$B$} & \multicolumn{2}{|c|}{$95 \% \mathrm{CI}$} & \multirow{2}{*}{$\beta$} & \multirow{2}{*}{$p$} & \multirow{2}{*}{$B$} & \multicolumn{2}{|c|}{$95 \% \mathrm{CI}$} & \multirow{2}{*}{$\beta$} & \multirow{2}{*}{$p$} \\
\hline & & lower & upper & & & & lower & upper & & & & lower & upper & & \\
\hline Men & -.01 & -0.10 & 0.08 & .00 & .858 & -.05 & -0.12 & 0.03 & -.03 & .241 & .01 & -0.15 & 0.18 & .00 & .888 \\
\hline \multicolumn{16}{|l|}{ Age } \\
\hline $20 \mathrm{~s}$ & .13 & -0.03 & 0.29 & .02 & .109 & .29 & 0.17 & 0.42 & .10 & $<.001$ & .00 & -0.31 & 0.32 & .00 & .978 \\
\hline 30s & .27 & 0.13 & 0.41 & .04 & $<.001$ & .25 & 0.14 & 0.35 & .10 & $<.001$ & .28 & -0.01 & 0.56 & .04 & .057 \\
\hline $40 \mathrm{~s}$ & .12 & -0.01 & 0.25 & .02 & .072 & .13 & 0.03 & 0.22 & .06 & .012 & .12 & -0.16 & 0.39 & .02 & .413 \\
\hline $50 \mathrm{~s}$ & .06 & -0.06 & 0.19 & .01 & .329 & .05 & -0.05 & 0.14 & .02 & .321 & .06 & -0.22 & 0.33 & .01 & .689 \\
\hline$>60 \mathrm{~s}$ (reference) & & & & & & & & & & & & & & & \\
\hline \multicolumn{16}{|l|}{ Educational background } \\
\hline junior high school/high school & .09 & 0.00 & 0.18 & .02 & .060 & .10 & 0.02 & 0.17 & .05 & .010 & .07 & -0.11 & 0.25 & .01 & .432 \\
\hline $\begin{array}{l}\text { specialized training college/ } \\
\text { two-year college } \\
\text { university/graduate school (reference) }\end{array}$ & -.02 & -0.13 & 0.08 & .00 & .649 & -.01 & -0.09 & 0.07 & .00 & .789 & -.05 & -0.24 & 0.14 & -.01 & .621 \\
\hline \multicolumn{16}{|l|}{ Employment } \\
\hline part-time, temporary worker & .15 & 0.04 & 0.26 & .02 & .009 & .02 & -0.08 & 0.11 & .01 & .739 & .26 & 0.06 & 0.47 & .04 & .013 \\
\hline self-employed & .02 & -0.15 & 0.19 & .00 & .848 & .00 & -0.13 & 0.13 & .00 & .977 & .00 & -0.33 & 0.33 & .00 & .981 \\
\hline unemployed & .05 & -0.09 & 0.19 & .01 & .473 & -.08 & -0.19 & 0.04 & -.03 & .202 & .14 & -0.11 & 0.39 & .02 & .265 \\
\hline full-time homemaker & .05 & -0.09 & 0.19 & .01 & .504 & -.05 & -0.16 & 0.06 & -.02 & .352 & .12 & -0.16 & 0.40 & .01 & .411 \\
\hline other & .13 & -0.05 & 0.30 & .01 & .152 & -.04 & -0.20 & 0.12 & -.01 & .589 & .24 & -0.06 & 0.54 & .02 & .114 \\
\hline \multicolumn{16}{|l|}{$\begin{array}{l}\text { Marital status } \\
\text { married (reference) }\end{array}$} \\
\hline not married & .01 & -0.12 & 0.14 & .00 & .869 & -.10 & -0.20 & 0.00 & -.05 & .052 & .12 & -0.12 & 0.36 & .02 & .340 \\
\hline bereaved & .22 & -0.08 & 0.51 & .01 & .152 & .02 & -0.20 & 0.24 & .00 & .857 & .55 & -0.06 & 1.16 & .02 & .076 \\
\hline divorced & .16 & -0.01 & 0.32 & .01 & .063 & .00 & -0.13 & 0.14 & .00 & .962 & .32 & 0.01 & 0.63 & .03 & .044 \\
\hline With child/children & -.12 & -0.23 & -0.01 & -.02 & .030 & -.07 & -0.16 & 0.01 & -.04 & .105 & -.18 & -0.39 & 0.04 & -.03 & .106 \\
\hline \multicolumn{16}{|l|}{ Household income } \\
\hline unknown & .09 & -0.03 & 0.22 & .01 & .155 & .05 & -0.05 & 0.15 & .02 & .309 & .20 & -0.05 & 0.46 & .03 & .120 \\
\hline$<$ JPY 400 million & .16 & 0.04 & 0.28 & .03 & .009 & .07 & -0.03 & 0.17 & .03 & .159 & .32 & 0.08 & 0.56 & .05 & .008 \\
\hline $\begin{array}{l}\text { JPY } 400-800 \text { million } \\
\text { >JPY } 800 \text { million (reference) }\end{array}$ & .03 & -0.08 & 0.14 & .01 & .580 & .02 & -0.06 & 0.10 & .01 & .597 & .10 & -0.12 & 0.33 & .02 & .357 \\
\hline Residential area: special alert area & -.07 & -0.15 & 0.01 & -.01 & .072 & -.01 & -0.07 & 0.05 & -.01 & .702 & -.13 & -0.27 & 0.02 & -.02 & .083 \\
\hline
\end{tabular}


Current psychiatric care

$\begin{array}{lllll}.17 & 0.04 & 0.29 & .02 & .008\end{array}$

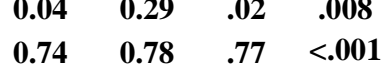

190.06

$\begin{array}{lll}06 & 0.32 & .05\end{array}$

$05 \quad .004$

$\begin{array}{lllll}.14 & -0.05 & 0.34 & .02 & .156\end{array}$

Suicidal ideation at $\mathbf{T 1}$

$\begin{array}{llll} & 0.82 & .71 & <.001\end{array}$

$\mathrm{CI}$ : confidence interval. 